\title{
Baptiste Bedessem
}
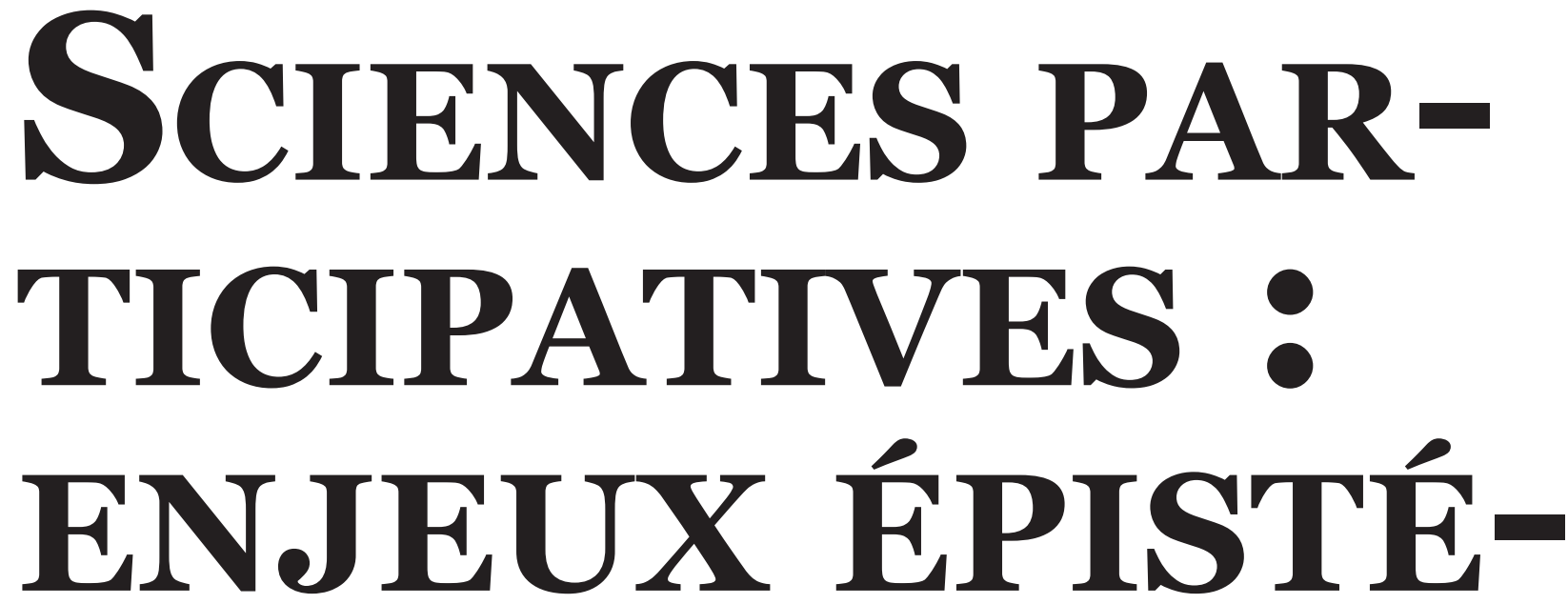

MOLOGIQUES

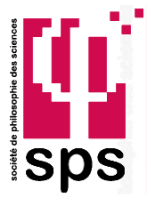

SOCIÉTÉ DE PHILOSOPHIE DES SCIENCES (SPS) 

MOLOGIQUES

\author{
Sommaire \\ 1 - Introduction \\ 2 - Les « sciences partici- \\ patives » : une diversité de \\ pratiques \\ 3 - Production des sa- \\ voirs dans les sciences parti- \\ cipatives \\ 4 - Quel rôle pour la philoso- \\ phie des sciences?
}

\begin{abstract}
La participation au développement scientifique et technique d'individus n'appartenant pas à la sphère académique est une pratique ancienne, qui connaît aujourd'hui un regain d'intérêt, notamment sous la forme de ce qu'il est convenu d'appeler " sciences participatives ». Les pratiques de participation se sont ainsi fortement diversifiées durant les deux dernières décennies dans des domaines comme les sciences de l'environnement, la biomédecine, l'astronomie ou encore la biologie fondamentale. Cette croissance marquée des programmes de «sciences participatives » a conditionné l'essor d'une littérature pluridisciplinaire s'attachant à décrire ces pratiques dans leurs dimensions sociologique, politique, éthique ou épistémologique. Cet état de l'art se propose de recenser les principaux enjeux d'ordre épistémologique posés par les différentes formes de participation citoyenne à la constitution de connaissances scientifiques, en se restreignant ici au cas des sciences de la nature. Il s'agira de souligner à la fois les opportunités et les obstacles qu'elles soulèvent tels qu'ils sont présentés et étudiés dans la littérature. L'un des objectifs de cette recension est de contribuer ainsi à constituer la question de la participation citoyenne comme un champ de recherche à part entière pour la philosophie des sciences.
\end{abstract} considerably diversified in the last decades, in fields such as environmental sciences, biomedicine, astronomy or fundamental biology. This growth of « citizen science » programs has generated a pluridisciplinary literature aiming at describing these practices in their sociological, political, ethical and epistemological dimensions. This state-of-the-art presents the main epistemological issues posed by citizen participation, in all its forms, to the constitution of scientific knowledge in the natural sciences. It highlights both the opportunities and the challenges created by citizen science, as they are presented and studied in literature. One of the objective of this contribution is then to help constituting the issue of citizen participation as a fully-fledged research field for philosophy of science.

Mots-clés : sciences participatives ; sciences citoyennes; sciences de l'environnement ; données massives ; épistémologie.

Keywords : participative science; citizen science; environmental sciences; big-data; epistemology.

\section{1 - Introduction}

La participation de chercheurs « amateurs », au sens d'acteurs n'appartenant pas aux communautés professionnelles dédiées à la recherche scientifique, à la constitution de savoirs intégrés au corpus savant est une pratique ancienne (Charvolin, Micoud et Nyhart 2007 ; Strasser et al. 2019). L'histoire des sciences de la nature, de la botanique du XIXe siècle $^{1}$ à l'écologie et aux sciences de la conservation contemporaines est ainsi particulièrement riche en interactions entre professionnels et amateurs ou profanes. Comme le rappellent notamment Prévot et al. (2016), les archives du Muséum d'Histoire Naturelle en témoignent sous la forme, par exemple, de ces Instructions pour les voyageurs et les employés dans les colonies sur la manière de recueillir, de conserver et d'envoyer les objets d'histoire naturelle, éditées par le Muséum au XIXe siècle. Ces brochures indiquent que les citoyens amateurs de science jouaient un rôle certain dans la collecte d' " objets d'histoire naturelle » provenant de régions lointaines, complétant ainsi le travail des naturalistes de l'époque. Alberti (2001) souligne également le rôle joué par les « clubs » naturalistes amateurs dans l'Angleterre victorienne, dans la description et la classification d'objets naturels (faune, flore, fossiles).

Cependant, malgré cet ancrage historique certain, les pratiques de participations citoyennes au développement scientifique et technique ont pris ces dernières décennies une ampleur inédite, tout en soulevant des enjeux nouveaux sur les plans éthique, politique et épistémologique.
Tout d'abord, on observe depuis quinze ans une croissance marquée non seulement du nombre de chercheurs engagés dans des programmes de sciences participatives mais aussi des publications scientifiques mobilisant les résultats de ces programmes - bien qu'en valeur relative, leur quantité, de l'ordre de $0.02 \%$ du total des publications, reste négligeable ${ }^{2}$ (Kullenberg et Kasperowski 2016). Cette dernière étude souligne également la croissance notable des travaux discutant les pratiques de participation sur les plans politiques, sociologiques, épistémologiques ou éthiques. Les raisons en sont multiples. D'une part, il faut noter que cette dynamique est largement portée par «l'impératif de participation » comme exigence centrale de nos démocraties contemporaines (Blondiaux 2008) et n'épargne pas la sphère scientifique. La participation est alors vue comme outil d'éducation ou d'émancipation des populations (Couvet et Prévot 2015). De manière moins normative, la sociologie a bien décrit le rôle croissant, assisté par la révolution numérique, joué par les « amateurs ", entre novices et professionnels, dans de nombreux domaines de la culture contemporaine (Flichy 2010). D'autre part, du point de vue de la science elle-même comme corpus de connaissances, la participation de citoyens non professionnels présente des intérêts certains : par exemple, en facilitant la collecte massive de données, elle est devenue un outil précieux pour les sciences de la conservation (Kosmala et al. 2016), bien que son utilisation - en dehors du champ de la simple collecte de données - reste limitée et débattue (Lewandowski et al. 2017).

Cette place croissante des sciences participatives dans le

1 - Comme le rappellent justement Haklay (2013) et Strasser et al. (2019), il est impropre de parler de " participation » d'amateurs avant le mouvement de professionnalisation de la science au cours du XIXe siècle. Plus exactement, ces auteurs soulignent que toute science était avant cela largement " amatrice », dans le sens où elle était pratiquée hors d'un cadre institutionnel et professionnel clair et bien différentié.

2 - aleur calculée par une étude menée sur la base des publications référencées sur la plateforme Web of Science (Kullenberg et Kasperowski 2016). 


\section{SCIENCES PARTICIPA- TIVES : ENJEUX ÉPISTÉ- MOLOGIQUES}

processus de recherche, ainsi que la prise de conscience de l'intérêt politique de ces pratiques (notamment comme outil de communication entre le champ scientifique et le reste du monde social) ont ainsi conditionné l'émergence récente d'une littérature variée qui interroge les caractéristiques et les conditions d'exercice de ces « sciences participatives » (voir Chlous et al. 2017 ; Strasser et al. 2019 pour des exemples récents). Notre contribution vise à établir un état de l'art des travaux portant sur les dimensions proprement épistémologiques de cette dynamique de participation dans toute la variété de ses manifestations : comment les « sciences participatives » sont-elles mobilisées par les chercheurs pour produire des savoirs ? Quelles en sont les limites ? Que peut-on penser des modalités institutionnelles de participation qui améliorent son impact sur le développement des connaissances scientifiques ? Les études dont nous proposons ici une recension s'intéressent, dans des visées descriptive et/ ou normative, à la manière dont la participation citoyenne contribue, et pourrait contribuer à l'avenir, à la constitution d'un corpus de savoir et d'expertises. Nous nous attachons ainsi à souligner tout à la fois les opportunités et les obstacles rapportés dans la littérature portant sur les « sciences participatives ». Cette littérature est particulièrement diverse et métissée. On y trouve ainsi des travaux de chercheurs en sciences de la nature concrètement engagés dans des programmes de participation, qui visent souvent à en dresser un bilan en termes de production de connaissances (fiabilité, impact sur le champ scientifique). Nous ferons également référence à de nombreuses contributions mobilisant des outils sociologiques qui proposent des études qualitatives ou quantitatives caractérisant les pratiques de participation (motivations des participants, perception des différents acteurs sur les programmes). Une littérature scientométrique, qui s'attache à mesurer et évaluer la production et la diffusion des savoirs issus de programmes de sciences participatives, s'est aussi développée. A cela s'ajoute enfin une littérature « grise » constituée de rapports institutionnels provenant des gouvernements ou d'organes supranationaux (OCDE, Commission européenne). Ces différentes sources bibliographiques constituent un complément nécessaire à la littérature plus directement rattachée à la philosophie des sciences. Elles offrent en effet des données empiriques précieuses permettant d'éclairer les pratiques de participation et d'en répertorier les principaux enjeux épistémologiques.

Nous débutons cet état de l'art par un tour d'horizon de la variété des programmes impliquant une participation citoyenne au développement scientifique et technique, ainsi que des principales typologies disponibles de ces pratiques (section 2). Nous présentons ensuite les principaux enjeux épistémologiques qui leur sont associés tels qu'ils sont mis en avant dans la littérature (section 3). Nous organisons alors notre propos en différenciant deux ordres de réflexion : la question du contrôle de la fiabilité des savoirs produits et de l'évaluation de leur impact d'une part ; celle des effets épistémologiques de la diversité (des valeurs, des intérêts, des positions sociales) des participants d'autre part. Ces enjeux seront exa- minés en tenant compte de la variété des pratiques de participation, depuis la collecte participative de données jusqu'à la co-construction des questions de recherche et des connaissances scientifiques entre professionnels et citoyens engagés. Comme nous le soulignons finalement dans la section IV, l'un des intérêts de cette recension est de contribuer à constituer la question de la participation citoyenne comme un champ de recherche fécond pour la philosophie des sciences. Notons dès à présent que nous nous restreignons, dans cet état de l'art, au cas des sciences de la nature : biologie, sciences de l'environnement, physique, biomédecine. Nous excluons donc de notre étude la question de la participation dans les sciences humaines (comme la sociologie ou la géographie) qui connaissent une histoire et des problématiques quelque peu distinctes à travers, par exemple, la « recherche-action » (Morrissette 2019).

\section{2 - Les « sciences participa- tives » : une diversité de pra- tiques}

2.1 Des sciences « par » et « pour » les citoyens Ce que la littérature francophone appelle communément « sciences participatives » ou « sciences citoyennes » correspond à l'anglais «citizen science $»^{3}$, terme utilisé originellement par le sociologue A. Irwin pour désigner l'expertise propre des individus, des groupes ou des communautés extérieurs au champ scientifique et tendant de fait à être exclus de la production des connaissances (Irwin 1995). Dans ce sens premier, l'expression citizen science est utilisée pour porter deux revendications complémentaires présentées ainsi par Cooper et Lewenstein (2016, p. 54) : " la science devrait se soucier des besoins et des problèmes rencontrés par les citoyens » et " le processus de production d'un savoir fiable doit être mené par les citoyens eux-mêmes. Les individus apportent à la science des savoirs locaux et contextuels et des contraintes géographiques, politiques et morales externes aux institutions scientifiques formelles ». Dans le premier cas, la participation concerne la formulation des problèmes et questions de recherche. Dans le second, elle touche aux différentes phases du processus de recherche lui-même : collecte des données, construction des résultats, production d'interprétations et d'hypothèses explicatives. Une science citoyenne se doit ainsi de prendre en compte à la fois les aspirations des différents publics constitutifs, dans la diversité de leurs intérêts et de leurs caractéristiques démographiques, du monde social et impliquer ces mêmes publics dans la production des savoirs ${ }^{4}$.

Les définitions forgées récemment pour la notion de « sciences participatives » recoupent largement cette double injonction. Le rapport Houllier, rendu en 2016 au ministre de l'Enseignement supérieur et de la Recherche désigne par ce vocable « des formes de production de connaissances scientifiques auxquelles des acteurs non-scientifiques profession-

3 - Terme pouvant lui-même être substitué, dans des contextes spécifiques, par une grande variété d'expressions, reflétant la diversité des pratiques de participation (Eitzel et al. 2017). En anglais, on trouve couramment, outre les termes « citizen science », les notions de " collaborative science », de " participatory research », de " humain sensing », de « collective intelligence », de « crowdsourcing », etc.

4 - En cela, le développement des sciences citoyennes recoupe largement les exigences contemporaines de " responsabilité » du développement scientifique et technique (Owen, Macnaghten et Stilgoe 2013 ; Rip 2016). 


\section{SCIENCES PARTICIPA- TIVES : ENJEUX ÉPISTÉ- MOLOGIQUES}

nels - qu'il s'agisse d'individus ou de groupes - participent de façon active et délibérée » (Houllier et Merilhou-Goudard 2016, p. 4). Pour le comité d'éthique du CNRS, l'expression désigne les "sciences auxquelles contribuent des citoyens amateurs à côté des professionnels de la recherche. On l'entend aussi comme les sciences ayant des vertus citoyennes au service de la société dans leur organisation et leur fonctionnement » (COMETS 2015, p. 2). On retrouve donc deux idées principales : (i) celle de la participation (à différents niveaux) des citoyens à la production des connaissances et (ii) celle de la mise au service de la science (des types de connaissances produites et de la manière dont elles sont produites) à la société (aux citoyens, aux groupes, aux communautés). Ces deux sens ont pu être contrastés comme " science contributive " (contributory science) et " science démocratique » (democratised citizen science), le sens original d'Irwin se rapprochant du second (Cooper et Lewenstein 2016 ; Eitzel et al. 2017).

Au-delà des définitions qu'on donne à ces concepts, il convient de reconnaître la grande diversité des pratiques qu'ils recouvrent, qui s'adaptent mal à la rigidité des catégorisations (Nascimento, Pereira et Ghezzi 2014 ; Heaton et al. 2016 ; Palacin-Silva et al. 2016). Comme le notait en 2013 un rapport de la Commission européenne portant sur les sciences participatives ${ }^{5}$ dans le domaine de l'environnement (Commission 2013), « le terme citizen science se réfère à une vaste panoplie de projets ayant des buts largement distincts, et des approches diverses dans leur travail avec les citoyens volontaires » (p. 4). En revanche, il faut cependant noter dès maintenant que la composition sociologique des groupes de participants semble peu diverse. Les enquêtes académiques ou institutionnelles menées sur ce sujet soulignent l'homogénéité de ces groupes de « citoyens volontaires » avec une surreprésentation des cadres d'une part et des retraités d'autre part (Dibner et Pandya 2018, p. 44). Outre leur haut niveau éducatif, les participants présentent aussi un intérêt initial pour les sciences, ou du moins pour l'objet de recherche en question (voir par exemple Palacin-Silva et al., 2016 dans le cas des observatoires de la biodiversité en Finlande). La section suivante liste quelques exemples de programmes récents dans le but d'illustrer la variété des pratiques de participation. Nous ordonnons ensuite ces références empiriques en présentant les principales typologies existantes.

\subsection{Quelques exemples}

Une étude scientométrique menée par Kullenberg et Kasperowski (2016) s'est attachée à caractériser les différents domaines faisant appel aux sciences participatives dans la construction des savoirs. Le plus large « est composé de recherches en biologie, en conservation et en écologie, et utilise les sciences participatives principalement comme une méthode de collecte et de classification de données » (p. 1). De manière générale, le contrôle ou le suivi de la biodiversité fait un usage croissant des sciences participatives (Prysby et Oberhauser 2004 ; Delaney et al. 2008 ; Crall et al. 2010 ; Danielsen et al. 2014 ; Chatzigeorgiou et al. 2016). Nous pouvons citer, en France, le réseau d'observatoires de la biodi- versité Vigie-Nature, porté depuis vingt ans par le Muséum National d'Histoire Naturelle et dédié au suivi des espèces communes sur l'ensemble du territoire (Cosquer, Raymond et Prévot-Julliard 2012 ; Couvet et Prévot 2015 ; Prévot et al. 2016 ; Prévot et al. 2018). Le rôle de la participation citoyenne dans la collecte des données sur l'environnement et la biodiversité a fait l'objet d'un rapport remis à la ministre de l'Écologie par Gilles Bœuf (président du Muséum) en 2012 (Bœuf, Allain et Bouvier 2012). Outre la collecte participative de données, il existe une forte tradition, dans les sciences environnementales, de mobilisation citoyenne ayant pour objectif d'interpeller la communauté scientifique et les pouvoirs publics sur des situations qui présentent un risque environnemental affectant des communautés particulières ou l'ensemble de la population (Miller-Rushing, Primack et Bonney 2012 ; Demeritt 2015 ; English, Richardson et Garzon-Galvis 2018) : nous pouvons citer par exemple la fracturation hydraulique (Willow et Wylie 2014 ; Kinchy 2016), la gestion des ressources naturelles (Yamamoto 2012) ou le risque nucléaire (Le Crosnier, Neubauer, Storup 2013). Un exemple relativement classique est celui des habitants de la localité de Diamond (Louisiane) qui ont décidé, au cours des années 1990, de construire une expertise scientifique concernant la pollution liée à une usine Shell voisine (Schrögel et Kolleck 2018). Les citoyens ont alors conçu des systèmes de collecte d'échantillons d'air atmosphérique qu'ils ont envoyés à des laboratoires professionnels pour analyse. Les habitants engagés dans cette démarche ont ensuite interprété les résultats tout en discutant les standards officiels utilisés pour évaluer les risques environnementaux.

Les champs de la biomédecine et de la santé publique regroupent aussi des cas bien connus de mobilisation de patients visant à influer sur les directions prises par la recherche (Den Broeder et al. 2018 ; pour la France, le groupe de réflexion avec les associations de malade de l'INSERM ${ }^{6}$ en est une bonne illustration). En biologie fondamentale, citons l'exemple du jeu sérieux Fold-it ayant pour objectif de prédire par tâtonnement, grâce à un ensemble de règles pré-établies, la structure de protéines données (Kelly et Maddalena 2015). Un article de 2011, publié dans Nature, rend compte de l'identification de la structure d'une protéine impliquée dans les infections par le VIH sur la base des résultats de ce programme (Khatib et al. 2011). En agronomie, dans le cadre de l'aide au développement, notons ici l'existence d'un programme de sélection participative d'espèces végétales impliquant des chercheurs de l'INRA et des agriculteurs pour la création de variétés adaptées aux agricultures à faibles intrants ou à l'agriculture biologique ${ }^{7}$.

Outre la biologie, la participation citoyenne est également bien représentée en astronomie. Un exemple souvent donné est celui de la recherche de la « planète 9 » menée par des volontaires à partir d'images fournies par la NASA. L'un des 


\section{SCIENCES PARTICIPA- TIVES : ENJEUX ÉPISTÉ- MOLOGIQUES}

programmes les plus populaires ces dernières années en astronomie est le projet « Galaxy Zoo », abrité par la plateforme «Zooniverse $»^{8}$. Il s'agit d'un programme de classification participative des galaxies sur la base d'images télescopiques (Watson et Floridi 2016) ${ }^{9}$. Face à la prolifération de programmes aux objectifs, méthodes et formes de participations variées, la littérature (travaux universitaires et rapports gouvernementaux) s'est attachée à dresser différentes typologies possibles, qui se recoupent assez largement.

\subsection{Typologies de la participation}

Selon Wilderman (2007), tout système de classification des sciences participatives doit se poser cinq questions : qui définit le problème ? Qui dresse le plan de l'étude ? Qui collecte les données ? Qui analyse ces données ? Qui les interprète? Suivant l'importance relative que l'on donne à chacune de ces perspectives, il est possible de dégager plusieurs stratégies de classification. Le rapport de la Commission européenne paru en 2013 et consacré à la participation citoyenne au développement scientifique et technique (Commission 2013) explicite alors trois types de classification possibles rappelées par Den Broeder et al. (2016) ${ }^{10}$. Ces classifications se fondent sur les phases du travail de recherche concernées par la participation, les objectifs poursuivis, ou le degré d'engagement.

\section{Phases du travail de recherche}

Il est tout d'abord possible de se focaliser sur les phases du travail de recherche dans lesquelles sont engagés les citoyens. Haklay (2013) propose ainsi de classer les sciences participatives (citizen science) en fonction du niveau de participation des citoyens au sein du processus de recherche. Ils distinguent ainsi : un niveau 1 , ou « crowdsourcing ", où les citoyens sont de simples « senseurs » (sensors) chargés de collecter des données ; un niveau 2, ou « intelligence distribuée " (distributed intelligence), où les citoyens sont des « interprètes basiques » (basic interpreters) (par exemple, en participant à des jeux sérieux à l'instar du programme fold-it cité plus haut); un niveau 3, ou " science participative » (participatory science), où les citoyens participent à la définition des problèmes à traiter ; et enfin un niveau 4, ou " science citoyenne totale » (extrem citizen science), où les citoyens sont engagés dans une véritable science collaborative, incluant la définition des problèmes, la collecte des données, et leur analyse. Le comité d'éthique du CNRS a proposé une classification du même type dans son avis sur les « sciences citoyennes » paru en 2015 (COMETS 2015).

\section{Objectifs poursuivis}

Certains auteurs classifient les sciences participatives en fonction des objectifs poursuivis (Wiggins et Crownston 2011) : l'action (les citoyens et les scientifiques collaborent pour résoudre des problèmes locaux) ; l'investigation (les programmes servent à traiter des questions strictement cognitives) ; et la conservation du bien commun (par exemple, la gestion collective des ressources naturelles et de la santé publique).

\section{Degré d'engagement}

Un troisième type de classification catégorise les sciences participatives en fonction du degré d'investissement ou d'engagement des participants. Cette stratégie, la plus répandue dans la littérature, conduit à croiser la question des phases du travail de recherche avec celle des objectifs poursuivis. Roy et al. (2012), repris par King et al. (2016), distinguent ainsi la science (i) " pour le public » (for the people), (ii) «par le public » (by the people), et (iii) « avec le public » (with the people). Dans le premier cas, les citoyens contribuent à la production des connaissances en étant conduits par des scientifiques qui définissent les objectifs et les protocoles de la recherche; dans le deuxième, les citoyens (les groupes ou communautés) proposent ou revendiquent des thèmes de recherche ; dans le troisième et dernier, il y a une forte coopération entre scientifiques et citoyens dans toutes les phases du processus de recherche. Une classification relativement similaire est proposée par Bonney et al. (2009a), qui distinguent trois types de « science citoyenne » (citizen science), qu'ils qualifient respectivement de (i) « projets contributifs »(contributory projects), dans lesquels les citoyens sont mobilisés pour réaliser des tâches relativement peu qualifiées, comme la collecte de données, sous la direction des scientifiques professionnels (pratique souvent qualifiée de crowdsourcing), (ii) " projets collaboratifs » (collaborative projects), au sein desquels les citoyens sont engagés dans des tâches cognitives plus exigeantes, incluant par exemple l'interprétation des résultats obtenus sur la base des données collectées, (iii) « projets co-créés » (co-created projects), où les citoyens collaborent étroitement avec les chercheurs dans la définition du problème à traiter et des méthodes à mettre en place.

En France, le rapport rendu en 2016 au ministre de la Recherche par F. Houllier utilise une classification assez proche des précédentes. Sur la base d'une analyse scientométrique, le rapport distingue alors trois grands types de sciences participatives :

a-Les sciences citoyennes désignent la contribution des citoyens amateurs à la collecte et à l'analyse des données. Le moteur en est surtout la curiosité individuelle, amplifiée par les outils numériques et le crowdsourcing. On en trouve surtout dans l'environnement, l'astrophysique et l'étude de la biodiversité.

b-La « recherche basée sur les communautés » (community based research) se fonde sur la collaboration entre des chercheurs et des groupes concernés pour diagnostiquer et résoudre des problèmes qui les affectent. Comme souligné dans la section précédente, on en trouve beaucoup d'exemples en santé publique (Den Broeder et al. 2016) et dans les sciences environnementales (Irwin

9 - D'autres exemples peuvent être trouvés en sciences humaines : géographie (Georis-Creuseveau, Noucher et Gourmelon 2018), linguistique (Svendsen 2018) ou sociologie, où la « recherche action participative » possède sa propre histoire (Morrissette 2019). Cependant, nous choisissons ici de nous focaliser sur le cas de la participation dans les sciences de la nature (biologie, sciences de l'environnement, physique), et les problèmes épistémologiques relativement nouveaux qu'elle pose.

10 -Notons que par souci de précision les termes anglais (majoritaires étant donné la domination de la littérature anglophone sur le sujet) dont la traduction pourrait induire une ambiguïté sont conservés dans leur langue originelle. 


\section{SCIENCES PARTICIPA- TIVES : ENJEUX ÉPISTÉ- MOLOGIQUES}

1995 ; Mielke, Vermabenc et Ellenbeck 2017 ; English, Richardson et Garzon-Galvis 2018). Ce type de science participative concerne typiquement des communautés soumises à un risque environnemental (souvent, une pollution locale), ou à une maladie spécifique (par exemple, une maladie génétique rare). Il s'agit donc de favoriser la capacitation (empowerment) des communautés se constituant autour d'un problème qui les affecte. Dans la description qu'en propose Houllier et Merilhou-Goudard (2016), il est possible de considérer cette community based research comme un cas particulier de co-created science au sens de Bonney et al. (2009a).

c-Les recherches participatives se rapprochent des précédentes, mais mettent plus en avant la notion de groupe de citoyens que celle de groupes concernés. Il s'agit surtout de relever de grands défis sociaux largement reconnus comme importants, dans une perspective d'innovation et de transformation sociale. De grandes organisations internationales, comme la Banque Mondiale, mettent souvent en place de telles démarches dans l'aide au développement ${ }^{11}$. En France, les projets de cultures participatives organisés par l'INRA (Institut National de Recherches Agronomiques) engagent les agriculteurs des pays en développement dans la recherche sur les ressources génétiques et leur utilisation dans les pratiques agronomiques ${ }^{12}$.

\section{Synthèse}

L'existence de ces différentes typologies permet de souligner la diversité des variables à prendre en compte pour caractériser empiriquement les programmes de sciences participatives. Schrögel et Kolleck (2018) ont ainsi proposé, dans un effort de synthèse, une méthode de classification identifiant trois dimensions pertinentes. La première concerne le pouvoir de décision délégué aux citoyens, depuis la simple « discussion publique » jusqu'à la « prise de décision publique ». Cette dimension est fortement inspirée des classifications décrivant les formes de participation citoyenne aux décisions dans un contexte démocratique, notamment celle d'Arnstein (1969). La seconde dimension se concentre sur le type d'implication cognitive et temporelle des participants. Cette implication dépend des phases du travail scientifique qui sont ouvertes à la participation. Par exemple, dans les programmes de crowdsourcing, l'implication est limitée à la phase de collecte de donnée. Les auteurs évoquent également la possibilité d'un apport du public à l'analyse (traitement des données), à l'interprétation (formulation de conclusions) et à la présentation des résultats. Ils notent en outre la possibilité d'une implication du public dans les phases précédentes du travail scientifique, amenant à la définition des problèmes de recherche et des questions à résoudre. La troisième dimension se penche sur le type de public concerné par la réalisation d'un projet de recherche donné. Par exemple, un programme peut être jugé important pour les scientifiques uniquement, pour les publics concernés ${ }^{13}$, ou pour l'ensemble de la société indistinctement.
De manière plus générale, la classification à privilégier dépend des objectifs que l'on se fixe. Dans notre cas, la présentation des différents types d'enjeux épistémologiques soulevés par les sciences participatives nécessite d'être attentif à la fois aux types de pratiques scientifiques dans lesquelles sont engagés les citoyens, et aux moteurs de la participation : est-elle conduite par une question posée spécifiquement par et pour les chercheurs, ou motivée par un problème collectif à résoudre ? Dans ce cadre, il nous semble que les classifications comme celle de Bonney et al. (2009a) ou celle proposée dans le rapport Houllier et Merilhou-Goudard (2016), basées sur le degré d'engagement des participants, sont particulièrement pertinentes. Comme nous l'avons vu, ces typologies reviennent en effet à considérer à la fois les phases du travail de recherche concernées par les pratiques de participation, et les objectifs qu'elles poursuivent. Dans le section II, lorsque cela s'avérera nécessaire à notre exposé, nous mobiliserons ainsi la distinction entre contributory, collaborative et co-created science (Bonney et al. 2009a).

\subsection{Bilan : des enjeux éthiques, politiques et épistémologiques}

Quelle que soit la forme qu'ils prennent, les programmes de science participative sont mis en valeur à la fois pour leur portée en termes de capacitation (empowerment) des populations (Commission 2014) ${ }^{14}$, d'éducation scientifique (Bonney et al. 2009a ; Cosquer, Raymond et Prévot-Julliard 2012 ; Prévot et al. 2018), mais également pour leur intérêt scientifique. Les pratiques de participation sont en effet, dans plusieurs domaines, motrices pour la production de savoirs nouveaux et de qualité, par exemple dans l'étude de la biodiversité (Couvet et al. 2008 ; Cosquer, Raymond et Prévot-Julliard 2012 ; Devictor et al. 2012 ; Scheliga et al. 2018). D'un autre côté, il est certain que l'irruption de publics extérieurs aux communautés scientifiques soulève des défis d'ordre éthiques, concernant par exemple la propriété des connaissances produites ou la rémunération des participants (Resnik, Elliott et Miller 2015 ; Heiss et Matthes 2017) ; politiques, touchant aux phénomènes d'exclusion spontanée des populations les plus vulnérables des processus de participation (Contamin, Legris et Spruyt 2017) ; et épistémologiques. Dans ce dernier cas, il convient en effet de noter l'existence d'une méfiance persistante des chercheurs vis-à-vis de la fiabilité des savoirs produits dans les projets impliquant une participation citoyenne, ce qui constitue un obstacle à l'utilisation plus massive des réseaux de volontaires, dans les sciences de la conservation notamment (Burgess et al. 2017 ; Law et al. 2017). Golumbic et al. (2017) ont ainsi mené une série d'entretiens avec des scientifiques ayant reçu des financements pour mener des programmes de recherche participative, afin de cerner leurs motivations et attentes. Ils montrent que bon nombre de scientifiques acceptent la participation avant tout pour obtenir des financements, mais éprouvent des difficultés à la tolérer tant sur le plan conceptuel (il leur semble difficile de considérer que la participation citoyenne puisse avoir

11 - Voir le rapport de la banque mondial consacré à ce sujet : http://documents.worldbank.org/curated/en/578241468765924957/pdf/multiopage.pdf

12 - http://participatory-plant-breeding.cirad.fr/

13 - Cette notion de «public concerné » ou «partie-prenante » (stakeholder) doit être comprise ici dans le sens classique qui lui est donnée dans la littérature sur la délibération publique (Kahane et Lopston 2013), comme désignant un groupe de personnes ayant un intérêt non-cognitif pour un problème donné, parce qüll est directement affecté par lui, ou quïl serait affecté par sa résolution.

14-Sur ce point, on pourra également se référer au site de l'European Citizen Science Association (https://ecsa.citizen-science.net/empowerment-inclusiveness-equity). 


\section{SCIENCES PARTICIPA- TIVES : ENJEUX ÉPISTÉ- MOLOGIQUES}

un effet bénéfique sur le plan épistémologique), que sur le plan pratique (les scientifiques préféreraient mener leurs études seuls). Une série d'entretiens du même type a été menée par Riesch et Potter (2014), qui notent également plusieurs réserves exprimées par les scientifiques quant à la pratique même de la participation, avec comme point central la question (légitime) de la qualité des données qui en sont issues (Engel et Voshell 2002 ; Kosmala et al. 2016). Le développement des sciences participatives rend donc nécessaire une réflexion systématique sur les différents défis qu'elles posent, et sur les opportunités qu'elles offrent pour la production de connaissances de qualité et utiles. La suite de cet état de l'art se propose d'établir une typologie raisonnée de la littérature, en forte croissance ces dernières années, traitant des multiples enjeux épistémologiques associés aux sciences participatives.

\section{3-Production des savoirs dans les sciences participatives}

Il nous semble que ces contributions peuvent être classées selon deux dimensions. La première regroupe des travaux qui se penchent, dans des visées descriptive et/ou normative, sur le contrôle de la fiabilité des connaissances produites (les données, les résultats, et leurs interprétations) et sur les possibilités d'évaluation de leur impact épistémique. La seconde interroge les effets de la diversité des valeurs, des intérêts, des positions sociales des participants sur la dynamique de production des connaissances.

\subsection{Contrôle et évaluation}

L'engagement de citoyens non professionnels dans la constitution de connaissances scientifiques est tout d'abord discuté en tant qu'il entre en tension avec les modes traditionnels de formation des chercheurs, du contrôle de la fiabilité des savoirs qu'ils produisent, et de l'évaluation de l'impact de ces productions.

\section{1.a Fiabilité des savoirs produits}

Un premier type d'enjeu épistémologique est lié à la fiabilité des savoirs produits, c'est-à-dire à leur conformité aux standards méthodologiques acceptés et utilisés au sein du champ scientifique, s'appliquant aussi bien à la collecte des données, à la construction des résultats, qu'à leur interprétation (Bonney et al. 2014 ; Hecker et al. 2018). La participation de citoyens non scientifiques pose alors question du fait de l'exigence souvent forte de prérequis théoriques et de capacités techniques qui caractérisent les pratiques de la science contemporaine. Ces savoirs conceptuels et pratiques sont en effet souvent nécessaires à la compréhension des questions de recherche, à l'exécution des protocoles, à l'analyse et l'interprétation des données et résultats. Or, les programmes de sciences participatives mobilisent des volontaires dont l'expertise est mal connue et souvent hétérogène (Wiggins et Crownston 2011 ; Gura 2013), conduisant dans certains cas à une large variabilité dans la qualité des données collectées par les différents participants (Crall et al. 2010).
Dans ce contexte, la littérature récente s'est penchée sur les multiples mécanismes mis en place pour assurer la fiabilité des savoirs produits par les programmes de sciences participatives. Un premier point important à relever est que le type de problèmes posés ici par la participation dépend étroitement des phases du travail de recherche dans lesquelles sont engagés les publics non scientifiques. Dans cette section, nous examinons donc séquentiellement les enjeux posés par les différents types de participation, en nous basant sur la classification classique de Bonney et al. (2009a), que l'on retrouve, comme nous l'avons vu, sous une forme proche dans le rapport Houllier et Merilhou-Goudard (2016).

\section{«Science contributive » (contributory science)}

Étant donné la domination des programmes du type " science contributive » (contributory science, Bonney et al. 2009a) dans le paysage des sciences participatives, un enjeu central est celui de la fiabilité des données collectées. Plusieurs travaux de synthèse proposent une recension des techniques mises en place par les chercheurs encadrant ces programmes pour en contrôler la qualité (Kosmala et al. 2016 ; Grand et al. 2016) et donc la crédibilité vis-à-vis de leurs utilisateurs potentiels (Freitag, Meyer et Whiteman 2016). Ces contributions insistent sur l'efficacité de ces contrôles, permettant la plupart du temps d'obtenir des jeux de données de haute qualité (voir Kosmala et al. 2016 et Parrish et al. 2018 pour des revues générales ; Danielsen et al. 2014 ; Herman-Mercer et al. 2018 pour des études de cas particulières). Précisons quelque peu la nature de ces mécanismes de contrôle, qui peuvent être classés selon trois dimensions.

La première de ces dimensions concerne la formation des participants, qui vise à leur apporter les connaissances techniques ou cognitives nécessaires à la réalisation d'un programme donné. Prysby et Oberhauser (2004) documentent, par exemple, le programme de formation des participants dans le cas du projet Monarch Larva Monitoring Project, visant à décrire les variations géographiques et temporelles, sur cinq ans et à l'échelle des États-Unis, de la répartition des larves de papillons Monarque. Il a été montré empiriquement que la présence d'une telle phase d'apprentissage permettait dans bien des cas aux participants de fournir des données de qualité comparable à celle des scientifiques professionnels (Fore, Paulsen et O'Laughlin 2001). Ces phases d'apprentissage sont souvent assorties d'une évaluation de la capacité des participants à réaliser les tâches qui leur sont assignées. C'est notamment le cas du programme « Galaxie Zoo », cité précédemment, qui fait usage d'une période probatoire durant laquelle les participants sont jugés sur leur capacité à classer correctement des images de galaxie de référence (Watson et Floridi 2016). Ce test est utilisé pour sélectionner les participants dont les résultats peuvent être jugés fiables. Cette question de la formation des participants soulève cependant des enjeux dépassant ces considérations techniques. Les interactions entre experts et citoyens volontaires ne peuvent être pensées sur le même mode que les relations classiques établies entre chercheurs chevronnés et étudiants ou chercheurs moins expérimentés. Le niveau 


\section{SCIENCES PARTICIPA- TIVES : ENJEUX ÉPISTÉ- MOLOGIQUES}

d'éducation scientifique préalable des volontaires est sans doute un facteur important à prendre en compte pour mettre en place des protocoles de formation adaptés. Cependant, peu de témoignages ont été publiés rendant compte des enjeux et difficultés spécifiques liés aux techniques d'apprentissage au sein des programmes de sciences participatives, la littérature se restreignant à des généralités sur les modes de formation : en classe, sur le terrain ou en ligne (voir par exemple Freitag, Meyer et Whiteman 2016).

Une deuxième famille de contributions interroge la manière dont sont élaborés et communiqués les protocoles de collecte par les chercheurs encadrant les programmes de science participative. L'enjeu est de mettre au point des modes de présentation des tâches à effectuer qui soient adaptés à leur utilisation par des citoyens non professionnels (Watson et Floridi 2016). Par exemple, Hochachka et al. (2012) rapportent le cas du programme « eBird » visant à répertorier les espèces d'oiseaux présentes sur un territoire donné : les fichiers de collecte de données sont pré-remplis par les scientifiques pour chaque espace géographique, et ne contiennent que les noms d'espèces les plus susceptibles d'y être présentes. Plusieurs études soulignent également la nécessité de garantir une grande adaptabilité de ces protocoles, qui doivent pouvoir être construits pas à pas, en interaction constante avec les participants (Crall et al. 2010). Brandon et al. (2003) ont ainsi montré, dans le cas d'un programme de recension d'espèces invasives dans certaines forêts des États-Unis, que les performances des participants variaient fortement en fonction des espèces à reconnaitre. Il est donc apparu nécessaire d'adapter ce programme de collecte, notamment en réservant certaines tâches aux participants les plus expérimentés.

Une troisième dimension, sans doute la plus richement explorée dans la littérature, concerne le contrôle ex-post de la qualité des données. L'ouverture, la transparence et la facilité de communication permise par l'utilisation d'outils numériques est ici un enjeu central (Resnik, Elliott et Miller 2015 ; Grand et al. 2016 ; Dias da Silva, Heaton et Millerand 2017). Par exemple, certains programmes utilisent des filtres automatiques afin de vérifier la cohérence interne des jeux de données collectées par chaque participant ( $\mathrm{Yu}$ et al. 2011). Le programme "Galaxie Zoo » teste la tendance de chaque participant à s'éloigner de la moyenne, et estime ainsi la fiabilité individuelle de leur contribution (Watson et Floridi 2016). Dans ce même programme, certains biais connus d'observation peuvent également être corrigés dans le traitement ex-post des données (Land et al. 2008). En écologie et dans les sciences de l'environnement, plusieurs programmes demandent aux participants de faire parvenir des photos, vidéos ou échantillons justifiant leur choix (par exemple, d'identification d'espèces), afin de vérifier la fiabilité de leurs données (Delaney et al. 2008). Notons enfin que bien souvent, ces trois dimensions (éducation et formation, adaptation des protocoles, et contrôle expost) sont développées conjointement par les chercheurs et les institutions encadrant les programmes de sciences participatives (Chatzigeorgiou et al. 2016).

\section{«Science collaborative » (collaborative science)}

Certains programmes demandent un engagement des participants dans d'autres phases du travail de recherche, par exemple dans le choix des sites de collecte de données, des méthodes utilisées, ou dans l'interprétation des résultats ( collaborative science »). Bonney et al. (2009a) donnent plusieurs exemples de précautions prises, dans ces cas, pour assurer la qualité et la fiabilité des données et des résultats issus des projets participatifs. Outre une période de formation approfondie précédant le programme, plusieurs initiatives proposent une session d'entretiens et de questionnaires afin d'évaluer les capacités techniques et cognitives acquises par les participants après la réalisation du projet : c'est le cas, par exemple, du projet Salal Harvest Sustainability Study, coordonné entre 2001 et 2004 par l'UC Berkeley, ayant pour objectif d'étudier l'effet de l'intensité de la récolte de Gaultheria shallon (palommier ou « Salal » en anglais) sur sa croissance (Ballard et Huntsinger 2006).

Ces programmes du type " collaborative science " possèdent également des qualités épistémiques intrinsèques, liées notamment à l'expertise locale des citoyens qui y sont engagés. De nombreuses études de cas devenues classiques ont pu montrer la valeur de ces expériences singulières, notamment dans le cas des sciences environnementales. L'exemple le plus connu est sans doute celui des moutons de Cumbria, développé par Wynne (1996), sur lequel nous ne reviendrons pas ici en détail. Le même type de dynamique s'observe dans le champ biomédical : l'expérience propre des patients, par exemple à propos des effets secondaires de leurs traitements, s'est souvent révélée positive pour la connaissance fondamentale des réactions biologiques (Godlee 2016).

Il est important de signaler, à ce stade, que la question du contrôle de la fiabilité des données et des résultats issus de ces programmes de science participative largement conduits et encadrés par des chercheurs professionnels est bien souvent un enjeu très technique, dépendant fortement des contextes précis de mise en œuvre des pratiques de participation.

\section{«Science co-crée » (co-created science)}

Les programmes de sciences participatives impliquant un engagement plus grand des citoyens dans la formulation même des questions de recherche (exemple de la « recherche basée sur les communautés » [community-based research] telle que définie dans le rapport Houllier et MerilhouGoudard 2016) posent un certain nombre de défis spécifiques. Il semble que deux situations peuvent être distinguées. Tout d'abord, la « co-created science » peut être impulsée par des communautés confrontées à des problèmes pratiques particuliers ; c'est souvent le cas, comme nous l'avons vu, dans les sciences environnementales (English, Richardson et Garzon-Galvis 2018) ou biomédicales. Dans ces cas-là, les citoyens engagés ont pour objectif de faire reconnaitre par la communauté scientifique et les institutions la réalité du risque encouru. En conséquence, ils s'engagent souvent dans une discussion critique, explicite et transparente, des méthodes ou des standards utilisés par les chercheurs professionnels. Citons ici l'exemple, donné dans la section I-2, de la collecte 


\section{SCIENCES PARTICIPA- TIVES : ENJEUX ÉPISTÉ- MOLOGIQUES}

et de l'analyse d'échantillons par les habitants de Diamond en Louisiane, dans le but de mettre en évidence la pollution de l'air liée à l'usine Shell voisine (Ottinger 2010 ; Schrögel et Kolleck 2018, p. 17). Un autre cas intéressant est celui des sociétés savantes constituées d'amateurs, posant et résolvant leurs propres questions de recherche, et qui là aussi sont en interaction constante avec les scientifiques professionnels, ce qui facilite la production de résultats jugés fiables (on pourra se référer au cas de la société entomologiste allemande ORION détaillé par Schrögel et Kolleck 2018).

Un second type de participation à la formulation et à la résolution de questions de recherche, bien que peut-être plus marginal, pose question quant à la fiabilité des résultats produits. Il s'agit des pratiques parfois désignées comme " piratage scientifique » (science hacking), qui se retrouvent surtout dans les sciences biologiques sous la forme des mouvements de "biohacking 》 ou de «do-it-yourself » (Ledford 2010 ; Meyer 2012). De manière générale, le but de ces initiatives est de bâtir un savoir en libre accès, en restant indépendant des institutions de la science professionnelle, qui sont parfois l'objet d'une certaine défiance. Cette promotion d'une " science de garage », se voulant parfois hors du contrôle des institutions scientifiques, pose une série de défis éthiques et politiques (Delfanti 2010). Dans une perspective épistémologique, un enjeu majeur est celui de la collaboration entre ces communautés de «hackers » et la science officielle (Landrain et al. 2013). En effet, l'une des caractéristiques de la science contemporaine comme entreprise collective est le contrôle mutuel des outils, protocoles et méthodes utilisés ; dans ce cadre, l'isolement d'une communauté en dehors de ces circuits de régulation est générateur de risques épistémiques, au sens de Biddle et Kukla (2017).

\section{1.b Évaluation de la recherche}

Une fois les savoirs produits, se pose naturellement la question des modalités de leur diffusion, d'une part, et de leur impact scientifique, d'autre part. De nombreuses publications soulignent la valeur épistémique des programmes de sciences participatives en mobilisant une série d'études de cas qui mettent en évidence la pertinence de ces connaissances pour le champ scientifique (Couvet et al. 2008 ; Bonney et al. 2009b ; Haywood 2013 ; Cox et al. 2015). Récemment, le besoin s'est fait sentir de procéder à des études bibliométriques plus systématiques, visant à identifier et classer les différents champs de recherche mobilisant les sciences participatives (Kullenberg et Kasperowski 2016 ; Bautista-Puig et al. 2019), ou à évaluer la capacité de ces programmes à générer des savoirs mobilisés par la suite au sein du champ scientifique (Tulloch et al. 2013 ; Follet et Strezov 2015), Ces études parviennent à dégager quelques tendances de fond, comme la forte croissance de l'usage de la participation notamment dans les sciences de la conservation, et leur implication dans des découvertes scientifiques d'importance (c'est-à-dire, ayant un impact notable sur le champ scientifique). Cependant, ces tentatives d'évaluation se heurtent à un certain nombre de difficultés, liées à la singularité des modes de pro- duction et de communication des savoirs impliquant la participation de publics non scientifiques. En particulier, il apparaît qu'une part non négligeable de ces connaissances ne sont pas diffusées sous la forme classique de publications évaluées par les pairs (Chandler et al. 2017). En outre, d'un point de vue plus technique, les publications issues des programmes de science participative ne sont pas aisément identifiables en tant que telles dans les bases de données bibliographiques comme Web of Science (Kullenberg et Kasperowski 2016). Les outils standards de la scientométrie, basées notamment sur la quantification de la dynamique de citations des articles, donnent ainsi une vision biaisée de l'impact des sciences participatives. Leur évaluation doit donc être enrichie par la prise en compte d'une plus grande diversité de critères. Wiggins et al. (2018) proposent dans ce cadre une grille d'analyse des productions scientifiques associées aux programmes de science participative, dans le but de guider la gestion et le financement de ces programmes. Leur « inventaire des productions scientifiques » (science products inventory), élaboré par un panel d'experts des sciences participatives, se propose de prendre en compte (i) les productions écrites, thèses, rapports et publications ; (ii) la taille des stocks de données, leur structure (existence de représentations visuelles, d'une documentation décrivant l'organisation des données) et leur mode de diffusion (par exemple, la fréquence de téléchargement); (iii) la communication des activités et des résultats (par exemple, l'organisation de conférences publiques, l'existence de sites web pour les projets, la diffusion régulière d'informations sous la forme de bulletins). L'ensemble de ces critères forme alors un espace multidimensionnel permettant de décrire et d'évaluer les programmes de science participative dans toute leur diversité.

\subsection{Participation et diversité(s) : enjeux épisté- mologiques}

La littérature proposant une analyse des sciences participatives dans une perspective épistémologique soulève une seconde grande famille d'enjeux, liés aux conséquences de la diversité sur la production des connaissances. En effet, la participation de publics non scientifiques à la production des savoirs a pour conséquence d'introduire une variété d'intérêts cognitifs et non cognitifs (pensons, par exemple, aux publics mobilisés pour résoudre des problèmes qui les affectent, en biomédecine ou en sciences del'environnement), mais également une pluralité de perspectives, de «points de vue » sur le monde social et naturel. Or, cette question du rôle de la diversité dans la production des connaissances s'est imposée ces dernières décennies comme centrale pour la philosophie des sciences (Steel et al. 2019). Elle regroupe plusieurs dimensions : diversité des valeurs, diversité des intérêts (sociaux et économiques), diversité démographique (ethnie, sexe, genre, position socio-économique...). Dans les faits, ces dimensions sont bien souvent associées : par exemple, notre appartenance à un groupe démographique donné détermine certains de nos valeurs non épistémiques et de nos intérêts socio-économiques. Cependant, d'un point 


\section{SCIENCES PARTICIPA- TIVES : ENJEUX ÉPISTÉ- MOLOGIQUES}

de vue épistémologique, elles portent chacune sur des enjeux qui leur sont propres. Par exemple, la question des intérêts non cognitifs pose celle de la neutralité des savoirs, au sens de Douglas (2009) repris par Koskinen (2018). Etre neutre en valeur (value-neutral) revient alors à ne pas utiliser volontairement certaines valeurs individuelles à la place des preuves empiriques. Classiquement, il est permis d'interroger cette neutralité des savoirs dans le cas où des conflits d'intérêts sont susceptibles de biaiser les résultats produits. La recherche industrielle, notamment pharmaceutique, est souvent ciblée sur ce point (Radder 2010). En revanche, cette exigence épistémique minimale ne revient pas à éliminer toute valeur non cognitive : comme l'a largement montré l'épistémologie sociale, une science totalement libre de valeurs n'est sans doute ni possible ni souhaitable (Rolin 2016). La mobilisation de valeurs non cognitives, par exemple sous la forme d' « hypothèses d'arrière-plan » (Longino 1990), est souvent inévitable dans les différentes phases du travail scientifique : formulation d'hypothèses, collecte de données, interprétation des résultats. Les arguments classiques liés au « risque inductif » (Rudner 1953 ; Douglas 2009), que nous ne rappellerons pas en détail ici, sont généralement utilisés pour justifier ce point. Afin de présenter de manière ordonnée les différents enjeux épistémologiques liés à cette question de la diversité dans les sciences participatives, nous proposons de distinguer trois types de contributions, interrogeant (a) la neutralité des savoirs, (b) la formulation des questions de recherche à traiter, et (c) la diversité des « points de vue » ou perspectives.

3.2.a Diversité des intérêts et neutralité des savoirs Dans de nombreux cas, les citoyens engagés dans des programmes de science participative se sont pas neutres vis-àvis des questions de recherche, c'est-à-dire qu'ils présentent un intérêt à sa résolution ou à sa non-résolution. Cela est bien évidemment le cas pour la « co-created science » (Bonney et al. 2009a), et notamment pour la « community-based research " (Houllier 2016) : par définition, les publics y sont directement impliqués en tant que « parties prenantes » (Kahane et Lopston 2013). Cependant, les formes plus modérées de participation sont en droit également concernées : comme nous l'avons vu dans les sections précédentes, il a été montré que la sociologie des groupes de participants en sciences de l'environnement est biaisée en faveur d'individus présentant un engagement particulier pour la préservation de la biodiversité (Palacin-Silva et al. 2016). Bien qu'il n'ait pas été avéré empiriquement que les intérêts singuliers des participants nuisent à la neutralité effective des savoirs produits, il existe une crainte répandue (notamment parmi les communautés scientifiques professionnelles) qu'ils influent sur la fiabilité des résultats (Golumbic et al. 2017 ; Jollymore et al. 2017). Les données pourraient ainsi être fabriquées, modifiées, falsifiées (Resnik, Elliott et Miller 2015 ; Nature 2015). Bien entendu, il faut noter que cette difficulté n'est pas restreinte aux sciences participatives, les scientifiques professionnels n'étant pas immunisés contre de potentiels conflits d'intérêts conduisant à des entorses à l'intégrité scientifique (Resnik 2010).
De manière plus significative en revanche, l'engagement de parties prenantes, explicitement concernées par un problème donné, dans le processus de recherche, est défendu par certains auteurs comme permettant une construction collective de la neutralité des savoirs produits. Par exemple, dans les sciences environnementales, certains groupes militants tendant à développer une expertise quant à la crédibilité des scientifiques vis-à-vis de leurs éventuels biais liés aux conflits d'intérêts auxquels ils peuvent se trouver confrontés. Yamamoto (2012) a mis en avant cette dynamique dans le cas d'une controverse autour de la gestion forestière. En conséquence, l'engagement de citoyens, même et surtout s'ils sont non neutres, semble encourager le développement d'une attitude de critique mutuelle vis-à-vis des manques possibles de neutralité des différents acteurs de la recherche (pour des études de cas allant dans ce sens, voir Cash et al. 2003 ; Barnes 2005; Parkins et Mitchell 2005 ; Elliott et al. 2017).

\section{2.b Diversité et construction participative des} questions de recherche

La participation citoyenne au développement scientifique et technique est susceptible d'influer sur la construction des objets et questions à traiter, en intégrant à l'agenda de recherche des problèmes formulés hors du champ scientifique. C'est notamment le cas de la co-created science ou de la community-based research (Houllier et Merilhou-Goudard 2016). Les modalités souhaitables de cette intégration n'ont été que rarement explorées par la philosophie. D'un point de vue épistémologique, une première dimension importante à prendre en compte ici interroge les liens qu'entretiennent les sciences «fondamentale » et « appliquée » ou « inspirée par l'usage » (Stokes 1997). Ces dernières années, des contributions se sont attachées à montrer le caractère positif, pour la production des savoirs, d'une intégration de questions pouvant être qualifiées d' " utilitaires » : en biologie moléculaire (Adam 2005 ; Adam, Carrier et Wilholt 2006 ; Bedessem 2018), en physique (Morrison 2011). Bien que ces études de cas apportent une série d'informations pertinentes pour penser les interactions épistémiques entre les différents types d'objectifs fixés à la recherche scientifique (construire des connaissances et servir le consommateur), il reste difficile de les mobiliser pour proposer une organisation générique et crédible de la participation citoyenne à l'orientation de l'effort de recherche. Dans ce contexte, certaines disciplines (et notamment, les sciences environnementales), visent cependant à développer, localement, des modalités de co-création de l'agenda de recherche adaptées à leurs caractéristiques et à leurs besoins propres (Cosquer, Raymond et Prévot-Julliard 2012).

Un second axe de réflexion exploré dans la littérature récente se penche sur l'implication d'une diversité de parties prenantes dans la formulation de questions de recherche portant sur des objets complexes présentant une multiplicité de dimensions politiques, économiques, sociales et scientifiques. 


\section{SCIENCES PARTICIPA- TIVES : ENJEUX ÉPISTÉ- MOLOGIQUES}

Des études de cas en sciences environnementales sont particulièrement mobilisées, dans le but de montrer comment des intérêts et des valeurs divergents conduisent à poser de manière distincte les problèmes à résoudre. Elliott (2011) développe ainsi l'exemple des études portant sur l'impact de certaines molécules utilisées par l'industrie agroalimentaire sur la santé, et montre comment les différentes parties prenantes (autorités sanitaires, ONG) déterminent a priori les doses limites dont la toxicité mérite d'être testée. Plus généralement, la diversité des parties prenantes tend à générer, sur un sujet donné, une multiplication d'expertises contradictoires (Tesh 1999 ; Eden, Donaldson et Walker 2006 ; Van der Vegt 2018). Intuitivement, cette diversité d'intérêts est positive sur le plan épistémologique, en ce qu'elle rend visible des questions de recherche nouvelles, ou des dimensions inexplorées de questions de recherche existantes (Harding 2015). Cependant, de manière quelque peu paradoxale, plusieurs auteurs ont également pu souligner qu'une telle prolifération d'avis et d'expertise portés par des groupes n'étant pas neutres vis-à-vis de l'objet de recherche en question peut être contre-productive d'un point de vue politique. C'est en particulier le cas dans les sciences de l'environnement (Sarewitz 2000, 2004), où la prolifération de savoirs parfois contradictoires portant sur des dimensions distinctes des controverses environnementales (parmi elles, la question du réchauffement climatique) peut avoir un effet paralysant sur la prise de décision publique. Dans une perspective plus directement épistémologique, la production participative des connaissances sur des sujets controversés peut également avoir des conséquences négatives sur le savoir produit. Cette dynamique de participation est en effet susceptible, d'une part, d'offrir une tribune à des positions scientifiques marginales et peu fondées. D'autre part, elle peut biaiser la manière dont les différentes dimensions du problème en question sont traitées. Biddle (2018) se penche par exemple sur les controverses autour de la culture des OGM, et montre que le manque de neutralité, associé aux différences de pouvoir et d'influence des groupes qui mandatent et utilisent des expertises scientifiques, freine la constitution d'un savoir rationnel et empiriquement fondé qui prendrait en compte de manière non partisane les différentes dimensions du débat. Dans ce contexte en effet, le développement et la visibilité des arguments, modèles et théories ne dépendent pas uniquement de leur pertinence épistémique, mais de l'activité et du pouvoir propres de groupes marqués idéologiquement ou politiquement. Les conditions politiques et sociales de la participation, dont l'étude dépend en grande partie d'un travail sociologique, jouent alors un rôle clé dans la détermination des caractéristiques épistémiques des savoirs produits (Chlous et al. 2017 ; Contamin, Legris et Spruyt 2017).

\section{2.c Diversité des « points de vue »}

Un troisième enjeu proprement épistémologique associé au rôle de la diversité est lié à la notion de «point de vue ». Ce thème regroupe des contributions plus théoriques en philo- sophie des sciences, tirant parti notamment du courant de " l'épistémologie des points de vue » (stand-point epistemology) dont Rolin (2016) propose une revue récente. Dans ce cadre, le lien entre participation, diversité et production des savoirs est classiquement établi de la manière suivante : dans les différentes phases du travail scientifique (formulation des hypothèses, mise au point des protocoles, collecte des données, interprétation des résultats et formulation des conclusions), des valeurs d'arrière-plan, qui peuvent dépendre du contexte social et culturel, jouent inévitablement un rôle (Douglas 2009). La position sociale d'un individu donné forge un point de vue spécifique sur le monde social et naturel, qui se traduit éventuellement par des hypothèses d'arrière-plan qui lui sont propres (ou propres à sa communauté). Dans ce cadre, la construction d'un savoir objectif ne peut être laissée à la discrétion d'une " communauté épistémique » donnée, fût-elle constituée de scientifiques professionnels, mais dépend d'une critique mutuelle entre des « épistémologies locales » portant des valeurs distinctes (Longino et Lennon 1997). Dans ce cadre, la participation de citoyens non scientifiques à la construction des savoirs est vue comme une opportunité d'améliorer leur objectivité, en facilitant l'identification et la critique des valeurs d'arrière-plan guidant les différentes phases du travail de recherche (Harding 2015).

Ces arguments sont bien étayés par l'histoire des sciences : les nombreuses études de cas proposées par l'épistémologie féministe, en premier chef, en montrent suffisamment la pertinence (Rolin 2016). Cependant, il n'est pas évident de comprendre comment le développement des sciences participatives peut assister la dynamique de critique mutuelle des hypothèses d'arrière-plan portées par des valeurs distinctes. Premièrement, cette conception sociale et interactionnelle de l'objectivité met en avant la nécessité d'une diversité au sein de la communauté scientifique. Quel niveau d'engagement des citoyens est alors à privilégier pour favoriser le travail de discussion des hypothèses d'arrière-plan ? Deuxièmement, il est possible que le type de diversité que l'épistémologie « du point de vue » défend, soulève certaines difficultés pratiques. Steel (2019) suggère ainsi que la diversité « démographique » (c'est-à-dire la diversité selon des critères démographiques, tels que l'ethnie, le genre, la classe) induit une forme de défiance entre les communautés épistémiques, défiance qui fait obstacle à la communication et donc au travail collaboratif entre agents épistémiques.

\section{4 - Quel rôle pour la philoso- phie des sciences?}

Pour clore cet état de l'art, il nous semble opportun de nous interroger sur les futurs rôles possibles que pourraient tenir la philosophie des sciences et l'épistémologie dans l'analyse descriptive et normative des conditions de la participation citoyenne au développement scientifique et technique. Cette 


\section{SCIENCES PARTICIPA- TIVES : ENJEUX ÉPISTÉ- MOLOGIQUES}

question des rôles possibles de la philosophie des sciences au XXIe siècle est très présente dans la littérature contemporaine (Giere 2003 ; Kourany 2003). Elle interroge tout à la fois les objets d'étude dont elle devrait se saisir, mais également les méthodologies à déployer : en particulier, l'usage croissant de méthodes qualitatives ou quantitatives inspirées des autres sciences humaines (Nersessian 2005 ; Wagenknecht, Nersessian et Andersen 2015 ; Svetlova 2015) brouille les frontières disciplinaires et interroge les spécificités de la démarche philosophique (Osbeck et Nersessian 2015 ; Mansnerus, Wagenknecht 2015 ; Thoren 2015). Ces débats ont de l'importance pour le sujet qui nous occupe, car comme nous avons pu le souligner dans cet état de l'art, la connaissance précise des modalités concrètes de la participation, qui dépend en grande partie d'un travail d'ordre sociologique, est cruciale pour juger des caractéristiques épistémologiques des sciences participatives. Prenant acte de ce contexte singulier, certains auteurs ont pu proposer un programme de recherche propre à la philosophie des sciences pour l'étude des pratiques de participation. Eigenbrode et al. (2007) et Elliott (2011) proposent ainsi qu'elle puisse jouer le rôle de facilitateur dans les délibérations entre experts et entre citoyens et experts. Reste encore à situer son action possible au sein des institutions existantes.

Une autre piste prend racine dans l'étude du rôle épistémique de la diversité. Comme nous l'avons souligné, l'une des caractéristiques des sciences participatives est en effet de promouvoir différentes formes de diversité dans les activités épistémiques : diversité de stratégies heuristiques, diversité de problèmes, de valeurs et d'intérêts, et ainsi que diversité démographique des agents épistémiques. Plusieurs atouts supposés des sciences participatives sont précisément liés à cette diversité : le manque de neutralité des communautés épistémiques engagées dans les programmes de sciences participatives pourrait favoriser l'adoption de perspectives critiques à propos des conflits d'intérêts possibles des différents acteurs ; la coexistence d'une diversité de problèmes et d'intérêts conduit à la production d'une variété d'expertises divergentes, dont l'existence pourrait être positive pour appréhender certains enjeux complexes ; la présence d'une diversité de valeurs contextuelles au sein des communautés épistémiques pourrait rendre possibles l'identification et la critique des hypothèses d'arrière-plan guidant la production du savoir scientifique. Cependant, plusieurs points restent en suspens. Il est permis notamment de se demander (i) quel type exactement de diversité est susceptible d'avoir des effets positifs sur le plan épistémique et (ii) quelles conditions institutionnelles sont requises pour promouvoir ces effets positifs. Il s'agit donc là, tout d'abord, d'un besoin de clarification conceptuelle à propos de la notion même de diversité, c'est-àdire à propos de la nature des groupes sociaux (scientifiques professionnels ou citoyens) et des objets (valeurs, intérêts, savoirs, position sociale) auxquels elle s'applique. Ensuite, se pose la question des modalités de communication entre des agents ou des communautés épistémiques portant des valeurs, intérêts ou positions sociales distincts (Wylie 2006). Comme Steel (2019) l'a noté, le processus « d'élaboration d'information ", décrivant la manière dont des propositions provenant de communautés épistémiques différentes peuvent ou non entrer dans des interactions fécondes, n'est pas encore assez étudié alors même que la diversité (telle qu'elle peut se manifester dans les programmes de participation citoyenne) est mise en valeur comme positive sur le plan épistémologique.

Une troisième voie de recherche possible a trait au développement de méthodes d'évaluation de la productivité des programmes de sciences participatives. Comme nous l'avons souligné, l'adaptation des techniques de scientométrie existantes pose un certain nombre de problèmes théoriques et pratiques. L'identification des paramètres importants à prendre en compte pour mesurer l'impact épistémologique (et politique) des sciences participatives nécessite sans doute un travail d'analyse conceptuelle dont la philosophie des sciences pourrait se saisir avec profit.

\section{Remerciements}

Ce travail a bénéficié du soutien du projet Idex Lyon Impulsion PartiSCiP ( Sciences participatives : nouvelles perspectives épistémologiques sur l'objectivité scientifique »). www. partiscip.com. 
REVUE

DE LA SOCIÉTÉ

DE PHILOSOPHIE DES SCIENCES

\section{RÉFÉRENCES}

ADAM, Matthias. 2005. Integrating Research and Development: the Emergence of Rational Drug Design in the Pharmaceutical Industry. Studies in History and Philosophy of Biological and Biomedical Sciences, 36, 513-537. Lien ADAM, Matthias, CARRIER, Martin, WILHOLT, Torsten. 2006. How to Serve the Customer and Still Be Truthful: Methodological Characteristics of Applied Research. Science and Public Policy, 33(6), 435-444. Lien

ALBERTI, Samuel J. M. M. 2001. Amateurs and professionals in one county: Biology and natural history in late victorian yorkshire. Journal of the History of Biology, 34(1), 115-147. Lien

ARNSTEIN, Sherry R. 1969. A ladder of citizen participation. Journal of the American Institute of planners, 35(4), 216224. Lien

BALLARD, Heidi L., HUNTSINGER, Lynn. 2006. Salal Harvester Local Ecological Knowledge, Harvest Practices and Understory Management on the Olympic Peninsula, Washington. Human Ecology, 34(4), 526-547. Lien

BARNES, Barry. 2005. The credibility of scientific expertise in a culture of suspicion. Interdisciplinary Science Reviews, 30(1), 1118. Lien

BAUTISTA-PUIG, Nuria, DE FILIPPO, Daniela, MAULEÓN, Elba, SANZ-CASADO, Elias. 2019. Scientific Landscape of Citizen Science Publications: Dynamics, Content and Presence in Social Media. Publications, 7(12), 1-22. Lien BEDESSEM, Baptiste. 2018. L'imprévisibilité de la science : un argument pour la liberté de recherche ? La découverte des ARNi comme étude de cas. Lato Sensu, 5(1), 37-43. Lien BIDDLE, Justin B. 2018. Antiscience zealotry? Values, epistemic risk, and the GMO debate. Philosophy of Science, 85(3), 360-379. Lien

BIDDLE, Justin B., KUKLA, Rebecca. 2017. The Geography of Epistemic Risk. In ELLIOTT, K. C., RICHARDS, T. (éds.). Exploring Inductive Risk: Case Studies of Values in Science. New York: Oxford University Press. 215-237.

BLONDIAUX, Luc. 2008. Le nouvel esprit de la démocratie. Actualité de la démocratie participative. Paris : Seuil.

BEUF, Gilles, ALLAIN, Yves- Marie, BOUVIER, Michel. 2012. L'apport des sciences participatives dans la connaissance de la biodiversité. Rapport remis à la ministre de l'Écologie. Lien

BONNEY, Rick, BALLARD, Heidi, JORDAN, Rebecca, McCALLIE, Ellen, PHILLIPS, Tina, SHIRK, Jennifer, WILDERMAN, Candie C. 2009a. Public Participation in Scientic Research. Washington, DC : Center for Advancement of Informal Science Education (CAISE).

BONNEY, Rick, COOPER, Caren, DICKINSON, Janis L, KELLING, Steve, PHILLIPS, Tina, ROSENBERG, Kenneth, SHIRK, Jennifer L. 2009b. Citizen Science: A Developing Tool for Expanding Science Knowledge and Scientific Literacy. BioScience, 59(11), 977-984. Lien

BONNEY, Rick, SHIRK, Jennifer, PHILLIPS, Tina, WIGGINS, Andrea, BALLARD, Heidi, MILLER-RUSHING, Abraham, PARRISH, Julia K. 2014. Next Steps for Citizen Science. Science, 343, 1436-1437. Lien

BRANDON, Alice, SPYREAS, Greg, MOLANO-FLORES, Brenda, CUNNIGHAM, Connie, ELLIS, James. 2003. Can volunteers provide reliable data for forest vegetation surveys?
SCIENCES PARTICIPATIVES : ENJEUX ÉPISTÉMOLOGIQUES
Nat Areas $J, 23,254-261$.

BURGESS, Hillary, DE BEY, Lauren, FROEHLICH, Halley, SCHMIDT, Natalaie, THEOBALD, Elinore, ETTINGER, Ailene, HILLE RIS LAMBERS, Janneke, TEWKSBURY, Josh, PARRISH, Julia. 2017. The science of citizen science: Exploring barriers to use as a primary research tool. Biological Conservation, 208, 113-120. Lien

CASH, David, CLARK, William, ALCOCK, Frank, DICKSON, Nancy, SELIN, Noelle, GUSTON, David, JÄGER, Jill. 2003. Knowledge systems for sustainable development. Proceedings of National Academy of Sciences, 100(14), 808691. Lien

CHANDLER, Mark, RULLMAN, Stan, COUSINS, Jenny, ESMAIL, Nafeesa, BEGIN, Elise, VENICX, Gitte, EISENBERG, Cristina, STUDER, Marie. Contributions to publications and management plans from 7 years of citizen science: Use of a novel evaluation tool on Earthwatchsupported projects. 2017. Biological Conservation, 28, 163173. Lien

CHARVOLIN, Florian, MICOUD, André, NYHART, Lynn. 2007. Des sciences citoyennes ? La question de l'amateur dans les sciences naturalistes. La Tour d'Aigues : Éditions de l'Aube.

CHATZIGEORGIOU, Georgios, FAULWETTER, Sarah, DAILIANIS, Thanos, SMITH, Vincent, KOULOURI, Panayota, DOUNAS, Costas, ARVANITIDIS, Christos. 2016. Testing the robustness of Citizen Science projects: Evaluating the results of pilot project COMBER. Biodiversity Data Journal, 4, e10859. Lien

CHLOUS, Frédérique, DOZIRE, Anne, GUILLAUD, Dominique, LEGRAND, Marine. 2017. Foisonnement participatif : des questionnements communs ? Natures Sciences Sociétés, 25(4), 327-335. Lien

COMETS. 2015. Avis du COMETS. Les sciences citoyennes. Comité d'éthique du CNRS. Lien

COMMISSION. 2013. Science for Environment Policy. Indepth-report: Environmental Citizen Science. Lien

COMMISSION. 2014. Green paper on citizen science for Europe: Towards a society of empowered citizens and enhanced research. Lien

CONTAMIN, Jean-Gabriel, LEGRIS, Martin, SPRUYT, Émilie. 2017. La participation de la société civile dans la recherche en matière d'environnement : les citoyens face au double sens caché participatif. Natures Sciences Sociétés, 25(4), 381-392. Lien

COOPER, Caren, LEWENSTEIN, Bruce. 2016. Two meanings of citizen science. In CAVELIER, D., KENNEDY, E. B. (éds.). The rightful place of science: Citizen Science. Tempe, AZ : Consortium for Science, Policy \& Outcomes. 51-61.

COSQUER, Alix, RAYMOND, Richard, PRÉVOT-JULLIARD, Anne-Caroline. 2012. Observation of Everyday Biodiversity: a New Perspective for Conservation? Ecology and Society, 17(4), 2. Lien

COUVET, Denis, JIGUET, Frédéric, JULLIARD, Romain, LEVREL, Harold, TEYSSEDRE, Anne. 2008. Enhancing citizen contribution to biodiversity science and public policy. Interdisciplinary Science Reviews, 33(1), 95-103. Lien COUVET, Denis, PRÉVOT, Anne-Caroline. 2015. Citizenscience programs: Towards transformative biodiversity governance. Environmental Development, 13, 39-45. Lien 
REVUE

DE LA SOCIÉTÉ

DE PHILOSOPHIE DES SCIENCES
SCIENCES PARTICIPATIVES : ENJEUX ÉPISTÉMOLOGIQUES
COX, Joe, OH, Ey, SIMMONS, Brooke, LINTOTT, Chris, MASTERS, Karen, GREENHILL, Anita, GRAHAM, Gary, HOLMES, Kate. 2015. Dening and measuring success in online citizen science: A case study of zooniverse projects. Computing in Science and Engineering, 17(4), 28-41. Lien CRALL, Alycia, NEWMAN, Greg, JARNEVICH, Catherine, STOHLGREN, Thomas, WALLER, Donald, GRAHAM, Jim. 2010. Improving and integrating data on invasive species collected by citizen scientists. Biol Invasions, 12, 3419-3428. Lien

DANIELSEN, Finn et al. 2014. A multicountry assessment of tropical resource monitoring by local communities. BioScience 64, 236-51. Lien

DELANEY, David, SPERLING, Corinne, ADAMS, Christiaan, LEUNG, Brian. 2008. Marine invasive species: validation of citizen science and implications for national monitoring networks. Biol Invasions, 10, 117-128. Lien

DELFANTI, Alessandro. 2010. Genome Hackers, rebel biology, open source and science ethic. Thèse de doctorat. Università degli studi di Milano.

DEMERITT, David. 2015. The promises of participation in science and political ecology. In PERREAULT, Tom, BRIDGE, Gavin, MCCARTHY, James (éds.). The Routledge Handbook of Political Ecology. Abingdon : Routledge. 224-234.

DEN BROEDER, Lea, DEVILEE, Jeroen, VAN OERS, Hans, SCHUIT, Albertine, WAGEMAKERS, Annemarie. 2018. Citizen science for public health. Health Promot. Int., 33(3), 505-514.

DEVICTOR, Vincent et al. 2012. Differences in the climatic debts of birds and butterflies at a continental scale. Nature Climate Change, 2(2), 121-124. Lien

DIAS DA SILVA, Patricia, HEATON, Lorna, MILLERAND, Florence. 2017. Une revue de littérature sur la "science citoyenne" : la production des connaissances naturalistes à l'ère numérique. Natures Sciences Sociétés, 25(4), 370-380. $\underline{\text { Lien }}$

DIBNER, Kenne, PANDYA, Rajul. 2018. Mapping the Landscape. In Learning Through Citizen Science: Enhancing Opportunities by Design. Washington : National Academies Press. 27-53.

DOUGLAS, Heather. 2009. Science, Policy and the ValueFree Ideal. Pittsburgh : University ofPittsburgh Press. Lien EDEN, Sally, DONALDSON, Andrew, WALKER, Gordon. 2006. Green groups and grey areas : Scientific boundarywork, nongovernmental organisations, and environmental knowledge. Environmental and Planning A, 38, 106-176. Lien

EIGENBRODE, Sanford, O'ROURKE, Michael, WULFHORST, J. D., ALTHOFF, David, GOLDBERG, Caren, MERRILL, Kaylani, MORSE, Wayde, NIELSEN-PINCUS, Max, STEPHENS, Jennifer, WINOWIECKI, Leigh, BOSQUEPÉREZ, Nilsa. 2007. Employing philosophical dialogue in collaborative science. BioScience, 57(1), 55-64. Lien EITZEL, Melissa, CAPPADONNA, Jennifer, SANTOS-LANG, Chris, DUERR, Ruth, VIRAPONGSE, Arika, WEST, Sarah, et al. 2017. Citizen science terminology matters: Exploring key terms. Citizen Science: Theory and Practice, 2(1), 1. Lien ELLIOTT, Kevin. 2011. Is a Little Pollution Good for You ? Incorporating Societal Values in Environmental Research. New York : Oxford University Press. Lien
ELLIOTT, Kevin, McCRIGHT, Aaron, SUMMER, Allen, DIETZ, Thomas. 2017. Values in environmental research: Citizens' views of scientists who acknowledge values. PLOS ONE, 12(10), e0186049. Lien

ENGEL, Sarah, VOSHELL, J. Reese. 2002. Volunteer biological monitoring: Can it accurately assess the ecological condition of streams? American Entomologist, 48(3),164177. Lien

ENGLISH, Paul, RICHARDSON, Maxwell, GARZONGALVIS, Catalina. 2018. From crowdsourcing to extreme citizen science: participatory research for environmental health. Annual review of public health, 39, 335-350. Lien FLICHY, P.atrice 2010. Le sacre de l'amateur. Sociologie des passions ordinaires l'ère numérique. Paris : Seuil.

FOLLETT, Ria, STREZOV, Vladimir. 2015. An analysis of citizen science based research: Usage and publication patterns. PLOS ONE, 10(11), e0143687. Lien

FORE, Leska, PAULSEN, Kit, O'LAUGHLIN, Kate. 2001. Assessing the performance of volunteers in monitoring streams. Freshwater Biology, 46(1), 109-123. Lien

FREITAG, Amy, MEYER, Ryan, WHITEMAN, Liz. 2016. Strategies Employed by Citizen Science Programs to Increase the Credibility of Their Data. Citizen Science: Theory and Practice, 1(1), 2. Lien

GEORIS-CREUSEVEAU, Jade, NOUCHER, Matthieu, GOURMELON, Françoise. De la production de données d'observation à l'usage d'informations géographiques naturalistes, Cybergeo: European Journal of Geography [En ligne], Science et Toile, document 878.

GIERE, Ronald. 2003. A new program for philosophy of science? Philosophy of Science, 70, 15-21. Lien

GODLEE, Fiona. 2016. At your next conference ask where the patients are. $B M J, 354$, i5123. Lien

GOLUMBIC, Yaela., ORR, Daniela, BARAM-TSABARI, Ayelet, FISHBAIN, Barak. 2017. Between vision and reality: A study of scientists views on citizen science. Citizen Science: Theory and Practice, 2(1), 1. Lien

GRAND, Ann, WILKINSON, Clare, BULTITUDE, Karen, WINFIELD, Alan. 2016. Mapping the hinterland: Data issues in open science. Public Understanding of Science, 25(1), 88103. Lien

GURA, Trisha. 2013. Citizen science: amateur experts. Nature, 7444(496), 259-261. Lien

HAKLAY, Muki. 2013. Citizen Science and Volunteered Geographic Information overview and typology of participation. In SUI, D.Z., ELWOOD, S., GOODCHILD M.F. (éds.) Crowdsourcing Geographic Knowledge: Volunteered Geographic Information (VGI) in Theory and Practice. 105122. Lien

HARDING, Sandra. 2015. Objectivity and Diversity: another Logic of Scientific Research. Chicago : Chicago University Press. Lien

HAYWOOD, Benjamin. 2013. A sense of place in public participation in scientific research. Science Education, 98(1), 64-83. Lien

HEATON, Lorna, MILLERAND, Florence, LIU, Xiao, CRESPEL, Elodie. 2016. Participatory science: encouraging public engagement in ONEM. International Journal of Science Education, Part B, 6(1), 1-22. Lien

HECKER, Susanne et al. 2018. Innovation in Citizen Science 
REVUE

DE LA SOCIÉTÉ

DE PHILOSOPHIE DES SCIENCES
SCIENCES PARTICIPA-

TIVES : ENJEUX ÉPISTÉMOLOGIQUES
- Perspectives on Science-Policy Advances. Citizen science: Theory and Practice, 3(1), 1-14. Lien

HEISS, Raffael, MATTHES, Jörg. 2017. Citizen science in the social sciences: A call for more evidence, Gaia, 26(1), 22-26. $\underline{\text { Lien }}$

HERMAN-MERCER, Nicole, ANTWEILER, Ronald, WILSON, Nicole, MUTTER, Edda, TOOHEY, Ryan, SCHUSTER, Paul. 2018. Data quality from a communitybased, water-quality monitoring project in the yukon river basin. Citizen Science: Theory and Practice, 3(2), 113. Lien HOCHACHKA, Wesley, FINK, Daniel, HUTCHINSON, Rebecca, SHELDON, Daniel, WONG, Weng-Keen, KELLING, Steve. 2012. Data-intensive science applied to broad-scale citizen science. Trends in Ecology \& Evolution, 27(2), 130137. Lien

HOULLIER, François, MERILHOU-GOUDARD, JeanBaptiste. 2016. Les sciences participatives en France : État des lieux, bonnes pratiques et recommandations. Rapport MESRI.

IRWIN, Alain. 1995. A Study of Citizen Expertise and Sustainable Development. London : Routledge.

JOLLYMORE, Ashlee, HAINES, Morgan, SATTERELD, Terre, JOHNSON, Mark. 2017. Citizen science for water quality monitoring: Data implications of citizen perspectives. Journal of Environmental Management, 200, 456-467. Lien KAHANE, David, LOPSTON, Kristjana. 2013. Stakeholder and citizen roles in public deliberation. Journal of public deliberation, 9(2),1-37.

KELLY, Ashley, MADDALENA, Kate. 2015. Harnessing agency for efficacy: Foldit and citizen science. Poroi, 11(1), 1-20. Lien

KHATIB, Firas. et al. 2011. Crystal structure of a monomeric retroviral protease solved by protein folding game players. Nature Structural and Molecular Biology, 18(10), 1175. Lien KINCHY, Abby. 2016. Citizen science and democracy: Participatory water monitoring in the marcellus shale fracking boom. Science as Culture, 26(1), 88-110. Lien

KING, Abby, et al. 2016. Leveraging citizen science and information technology for population physical activity promotion. Journal of the American College of Sports Medicine, 1, 3044.

KOSKINEN, Inkeri. 2018. Defending a risk account of scientic objectivity. The British Journal for the Philosophy of Science. Lien

KOSMALA, Magaret, WIGGINS, Andrea, SWANSON, Alexandra, SIMMONS, Brooke. 2016. Assessing data quality in citizen science. Frontiers in Ecology and the Environment, 14(10), 551-560. Lien

KOURANY, Janet. 2003. A philosophy of science for the twenty-first century. Philosophy of Science, 70(1), 1-14. Lien KULLENBERG, Christopher, KASPEROWSKI, Dick. 2016. What is citizen science? A scientometric meta-analysis. PLoS ONE, 11(1), e0147152. Lien

LAND, Kate. et al. 2008. Galaxy Zoo: The large-scale spin statistics of spiral galaxies in the Sloan Digital Sky Survey. Monthly Notices of the Royal Astronomical Society, 388(4), 1686-1692. Lien

LANDRAIN, Thomas, MEYER, Morgan, PEREZ, Ariel, REMI, Sussan. 2013. Do-it-yourself biology: challenges and promises for an open science and technology movement. Syst
Synth Biol, 7, 115-126. Lien

LAW, Edith, GAJOS, Krzysztof, WIGGINS, Andrea, GRAY, Mary, WILLIAMS, Alex. 2017. Crowdsourcing as a tool for research: Implications of uncertainty. Proceedings of the 2017 ACM Conference on Computer Supported Cooperative Work and Social Computing. 1544-1561. Lien

LE CROSNIER, Hervé, NEUBAUER, Claudia, STORUP, Bérangère. 2013. Sciences participatives ou ingénierie sociale : quand amateurs et chercheurs co-produisent les savoirs. Hermès, La Revue, 67, 68-74. Lien

LEDFORD, Heidi. 2010. Life hackers. Nature, 467, 650-652. $\underline{\text { Lien }}$

LEWANDOWSKI, Eva, CALDWELL, Wendy, ELMQUIST, Dane, OBERHAUSER, Karen. 2017. Public perceptions of citizen science. Citizen Science: Theory and Practice, 2(1), 19. Lien

LONGINO, Helen. 1990. Science as Social Knowledge. Princeton : Princeton University Press.

LONGINO, Helen, LENNON, Kathleen. 1997. Feminist Epistemology as a Local Epistemology. Proceedings of the Aristotelian Society, Supplementary Volumes, 71, 19-35. Lien MANSNERUS, Erika, WAGENKNECHT, Susann. 2015. Feeling with the Organism: A Blueprint for an Empirical Philosophy of Science. In WAGENKNECHT, S., NERSESSIAN, N. J., ANDERSEN, H. (éds.). Empirical philosophy of science: Introducing qualitative methods into philosophy of science. Cham : Springer. 37-61. Lien

MEYER, Morgan. 2012. Build your own lab: Do-it-yourself biology and the rise of citizen biotecheconomies. Journal of Peer Production, 2, 1-4.

MIELKE, Jahel, VERMABENC, Hannah, ELLENBECK, Saskia. 2017. Ideals, practices, and future prospects of stakeholder involvement in sustainability science. PNAS, E10648E10657. Lien

MILLER-RUSHING, Abraham, PRIMACK, Richard, BONNEY, Rick. 2012. The history of public participation in ecological research. Frontiers in Ecology and Evolution, 20(6), 285-290. Lien

MORRISON, Margaret. 2011. Between the Pure and Applied: The Search for the Elusive Middle Ground. In CARRIER M., NORDMANN, A. (éds.). Science in the Context of Application. Boston : Boston Studies in the Philosophy of Science, vol. 274. 31-45. Lien

MORRISSETTE, Joëlle. 2019. Recherche-action et recherche collaborative. Quel rapport aux savoirs et à la production de savoirs ? Nouvelles pratiques sociales, 25(2), 35-49. Lien

NASCIMENTO, Susana, GUIMARAES PEREIRA, Angela, GHEZZI, Alessia. 2014. From citizen science to do it yourself science. An annotated account of an on-going movement. Luxembourg : Publications of the European Union.

NATURE. 2015. Editorial: Rise of the citizen scientist. Nature, 524, 265. Lien

NERSESSIAN, Nancy. 2005. Interpreting scientic and engineering practices: integrating the

cognitive, social, and cultural dimensions. In GORMAN, M.E., TWENEY, R.D., GOODING, D.C., KINCANNON, A. P. (éds.). Scientific and Technological Thinking. Mahwah : L. Erlbaum. 17-56.

OSBECK, Lisa, NERSESSIAN, Nancy. 2015. Prolegomena to an Empirical Philosophy of Science. 
REVUE

DE LA SOCIÉTÉ

DE PHILOSOPHIE DES SCIENCES
SCIENCES PARTICIPATIVES : ENJEUX ÉPISTÉMOLOGIQUES
In WAGENKNECHT, S., NERSESSIAN, N. J., ANDERSEN, H. (éds.). Empirical philosophy of science: Introducing qualitative methods into philosophy of science. Cham : Springer. 13-36. Lien

OTTINGER, Gwen. 2010. Buckets of resistance: Standards and the effectiveness of citizen science. Science, Technology \& Human Values, 35(2), 244-270. Lien

OWEN, Richard, MACNAGHTEN, Phil, STILGOE, Jack. 2013. Responsible research and innovation: From science in society to science for society, with society. Science and Public Policy, 39(6), 751-760. Lien

PALACIN-SILVA, Victoria, SEFFAH, Ahmed, HEIKKINEN, Karl, PORRAS, Jari, PYHALAHTI, Timo, SUCKSDOR, Yrjö, ANTTILA, Saku, ALASALMI, Hanna, BRUUN, Eeva, JUNTTILA, Sofia. 2016. State-of-the Art Study in Citizen Observatories: Technological Trends, Development Challenges and Research Avenues. Reports of the Finnish Environment Institute, 28.

PARKINS, John, MITCHELL, Ross. 2005. Public participation as public debate: A deliberative turn in natural resource management. Society and Natural Resources, 18, 52940. Lien

PARRISH, Julia, BURGESS, Hillary, WELTZIN, Jake, FORTSON, Lucy, WIGGINS, Andrea, SIMMONS, Brooke. 2018. Exposing the Science in Citizen Science: Fitness to Purpose and Intentional Design. Integrative and Comparative Biology, 58(1), 150-160. Lien

PRÉVOT, Anne-Caroline, DOZIÈRES, Anne, TURPIN, Sébastien, JULLIARD, Romain. 2016. Les réseaux volontaires d'observateurs de la biodiversité (vigie-nature) : quelles opportunités d'apprentissage ? Cahiers de l'action, 47, 35-40. Lien

PRÉVOT, Anne-Caroline, CHEVAL, Hélène, RAYMOND, Richard, COSQUER, Alix. 2018. Routine Experiences of Nature in Cities Can Increase Personal Commitment Toward Bidiversity of Conservation. Biological Conservation, 226,18. Lien

PRYSBY, Michelle, OBERHAUSER, Karen. 2004. Temporal and geographic variation in monarch densities: citizen scientists document monarch population patterns. In OBERHAUSER, Karen S., SOLENSKY, M.J. (éds). Monarch Butterfly Biology \& Conservation. Ithaca : Cornell University Press.

RADDER, Hans. 2010. The Commodification of Academic Research: Science and the Modern University. Pittsburgh : University of Pittsburgh Press. Lien

RESNIK, David. 2010. Financial Interests and the Norms of Academic Science. In RADDER H. (éd.). The Commodification of Academic Research: Science and the Modern University. Pittsburgh : University of Pittsburgh Press. 65-89. Lien

RESNIK, David, ELLIOTT, Kevin, MILLER-RUSHING, Abraham. 2015. A framework for addressing ethical issues in citizen science. Environmental Science and Policy, 54, 47581. Lien

RIESCH, Hauke, POTTER, Clive. 2014. Citizen science as seen by scientists: Methodological, epistemological and ethical dimensions. Public Understanding of Science., 23(1), 107-120.

RIP, Arie. 2016. The clothes of the emperor. An essay on RRI in and around Brussels. Journal of responsible innovation,

\section{3(3), 290-304. Lien}

ROLIN, Kristina. 2016. Values, standpoints, and scientific/ intellectual movements. Studies in History and Philosophy of Science, 56, 11-19. Lien

ROY, Helen, POCOCK, Michael, PRESTON, Chris, ROY, David, SAVAGE, Joanna, TWEDDLE, John. 2012. Understanding Citizen Science \& Environmental Monitoring. Final Report on behalf of UK-EOF.

RUDNER, Richard. 1953. The scientist qua scientist makes value judgments. Philosophy of Science, 20(1), 1-6. Lien

SAREWITZ, Daniel. 2000. Science and Environmental Policy: An Excess of Objectivity. In FRODEMAN, Robert (éd.). Earth Matters : The Earth Sciences, Philosophy, and the Claims of Community. Upper Saddle River : Prentice Hall.

SAREWITZ, Daniel. 2004. How science makes environmental controversies worse. Environmental Science and Policy, 7(5), 385403. Lien

SCHELIGA, Kaja, FRIESIKE, Sascha, PUSCHMANN, Cornelius, FECHER, Benedikt. 2018. Setting up crowd science projects. Public Understanding of Science, 27(5), 515-534. Lien

SCHRÖGEL, Philipp, KOLLECK, Alma. 2018. The Many Faces of Participation in Science: litterature Review and Proposal for a Three-Dimensional Framework. Science \& Technology Studies, 32(2), 77-99. Lien

STEEL, Daniel, FAZELPOUR, Sina, CREWE, Bianca, GILLETTE, Kinley. 2019. Information elaboration and epistemic effects of diversity, Synthese. Lien

STOKES, Donald. 1997. Pasteur's Quadrant. Basic Science and Technological Innovation. Washington : Brookinks Institution Press.

STRASSER, Bruno, BAUDRY, Jérôme, MAHR, Dana, SANCHEZ, Gabriela, TANCOIGNE, Elise. "Citizen Science”? Rethinking Science and Public Participation. 2019. Science \& Technology Studies, 32(2), 52-76. Lien

SVENDSEN, Bente. 2018. The dynamics of citizen sociolinguistics. Journal of Sociolinguistics, 22(2), 137-160. $\underline{\text { Lien }}$

SVETLOVA, Ekaterina. 2015. The Benets and Challenges of Qualitative Methods. In WAGENKNECHT, S., NERSESSIAN, N. J., ANDERSEN, H. (éds.). Empirical philosophy of science: Introducing qualitative methods into philosophy of science. Cham : Springer. 37-61.

TESH, Sylvia. 1999. Citizen experts in environmental risk. Policy Sciences, 32, 3958. Lien

THOREN, Enrik. 2015. History and Philosophy of Science as an Interdisciplinary Field of Problem Transfers. In WAGENKNECHT, S., NERSESSIAN, N. J., ANDERSEN, H. (éds.). Empirical philosophy of science: Introducing qualitative methods into philosophy of science. Cham : Springer. 127-143. Lien

TULLOCH, Ayesha, POSSINGHAM, Hugh, JOSEPH, Liana, SZABO, Judith, MARTIN, Tara. 2013. Realising the full potential of citizen science monitoring programs. Biological Conservation, 165, 128-138. Lien

VAN DER VEGT, Rens. 2018. A literature review on the relationship between risk governance and public engagement in relation to complex environmental issues. Journal of Risk Research, 21(11), 1-18. Lien 


\section{SCIENCES PARTICIPA- TIVES : ENJEUX ÉPISTÉ- MOLOGIQUES}

WAGENKNECHT, Susann, NERSESSIAN, Nancy, ANDERSEN, Hanne. 2015. Empirical philosophy of science: Introducing qualitative methods into philosophy of science. Cham : Springer. Lien

WATSON, David, FLORIDI, Luciano. 2016. Crowdsourced science: sociotechnical epistemology in the e-research paradigm. Synthese, 195(2), 741-764. Lien

WIGGINS, Andrea, CROWNSTON, Kevin. 2011. From conservation to crowdsourcing: A typology of citizen science. Hawaii : International Conference on System Sciences. Lien WIGGINS, Andrea, BONNEY, Rick, LEBUHN, Gretchen, PARRISH, Julia, WELTZIN, Jake. 2018. A Science Products Inventory for Citizen-Science Planning and Evaluation. BioScience, 68(6), 436-444. Lien

WILDERMAN, Candie. 2007. Models of Community Science: Design Lessons from the Field. Citizen Science Toolkit Conference, Ithaca.

WILLOW, Anna, WYLIE, Sara. 2014. Politics, ecology, and the new anthropology of energy: exploring the emerging frontiers of hydraulic fracking. Journal of Political Ecology, 21, 222-236. Lien

WYLIE, Alison. 2006. When Difference Makes A Difference: Epistemic Diversity and Dissent. Episteme: Journal of Social Epistemology, 3(1), 1-7. Lien

WYNNE, Brian. 1996. May the Sheep Graze? A reflexive view of the expert-lay knowledge divide. In LASH, S., SZERSZYNSKI, B., WYNNE, B. (éds.). Risk, Environment and Modernity, p.44-84. London : Sages Publication. YAMAMOTO, Yuri. 2012. Values, objectivity and credibility of scientists in a contentious

HISTORIQUE

Article initialement soumis le 5 août 2019.

Article révisé soumis le 21 octobre 2019.

Article accepté le 24 octobre 2019.

SITE WEB DE LA REVUE

ojs.uclouvain.be/index.php/latosensu

ISSN 2295-8029

DOI http://dx.doi.org/10.20416/LSRSPS.V7I1.1

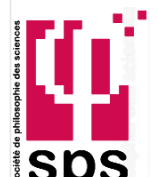

SOCIÉTÉ DE PHILOSOPHIE DES SCIENCES (SPS) École normale supérieure 45, rue d'Ulm 75005 Paris www.sps-philoscience.org natural resource debate. Public Understanding of Science, 21(1), 101-125.

YU, Jun, KELLING, Steve, GERBRACHT, Jeff, WONG, WengKeen. 2011. Emergent filters: automated data verification in a large-scale citizen science project. In IEEE Seventh International Conference on e-Science, 5 th-8th December. Stockholm : Workshop Proceedings. 20-27. Lien

\section{CONTACT ET COORDONNÉES}

Baptiste Bedessem

Università Ca'Foscari

baptiste.bedessemp@gmail.com

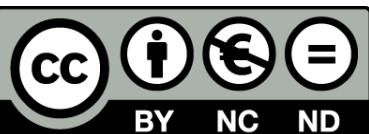

